\title{
End-of-life and goals of care discussions with cancer patients in the coronavirus pandemic
}

\section{Essay/Personal Reflection}

Cite this article: Mott FE, Bruera E, Johnson F (2021). End-of-life and goals of care discussions with cancer patients in the coronavirus pandemic. Palliative and Supportive Care 19, 384-385. https://doi.org/ $10.1017 /$ S1478951520000668

Received: 11 June 2020

Revised: 20 July 2020

Accepted: 20 July 2020

Author for correspondence: Frank Mott, Thoracic, Head and Neck Medical Oncology, University of Texas M.D. Anderson Cancer Center, 1515 Holcombe, Unit 432, Houston, TX 77030, USA. E-mail: fmott@mdanderson.org
Frank E. Mott, M.D. F.A.C.P. ${ }^{1}$ D , Eduardo Bruera, M.D. ${ }^{2}$ and Faye Johnson, M.D. PH.D. ${ }^{3}$

${ }^{1}$ Thoracic, Head and Neck Medical Oncology Department, University of Texas MD Anderson Cancer Center, Houston, TX; ${ }^{2}$ Department of Palliative, Rehabilitation, and Integrative Medicine, University of Texas MD Anderson Cancer Center, Houston, TX and ${ }^{3}$ Graduate School of Biomedical Sciences, Thoracic, Head and Neck Medical Oncology Department, University of Texas MD Anderson Cancer Center, Houston, TX

Advances in the treatment of cancer have improved both the quantity and quality of life for patients in recent years. Better supportive care, improved therapies to moderate side effects and symptom management, the understanding of molecular mechanisms, and the advent of targeted agents and immunotherapy have all improved the outcome for many cancer patients. Yet, the sad reality is that most patients will eventually succumb to their disease.

Honest discussions with patients and their families to outline realistic goals of care (GOC) and, when appropriate, end-of-life (EOL) care are important. Unfortunately, for many years and even still today, these conversations have been minimized or even omitted. There are many explanations for this deficiency. Training in palliative care and delivery of bad news is often a minor part of the education program for many clinicians, even oncologists (Baile, 1999; Ambuel, 2001; Van de Kieft, 2001; Rosenbaum, 2004; Back 2005; Marcus et al., 2006). Electronic records and computer-based treatment algorithms have reduced the amount of face-to-face personal interactions so vital to understanding the emotions of cancer and patient/family dynamics. New therapies, while advancing survival, have caused patients and clinicians to feel that there are still potential "curative" or disease-remitting options, even after multiple lines of futile therapy. Palliative care and EOL conversations, especially if brought up late in the course of a patient's disease, after multiple different treatments, may be seen as "giving up."

These conversations are difficult and uncomfortable. As a result, they are often deferred for a later appointment, or to a colleague or Palliative Care specialist. Conversations at the EOL are often bungled and not done well (Marcus et al., 2006; Marcus and Mott, 2013, 2014; Mott and Adams, 2019). While many training programs include palliative care modules, there is much variability across different programs as well as different levels of awareness and beliefs among trainees in different specialties (Wong et al., 2016; Reddy et al., 2019). Patients' and families' perceptions of what palliative care means and its larger concept of GOC and EOL discussions are additional hurdles (Lo et al., 1999; Singer et al., 1999; Heyland, 2006). Even though the addition of palliative care along with the cancer therapy has been shown to improve outcomes (Mott and Adams, 2019; Temel et al., 2010), many clinicians fail to enlist palliative or supportive care teams until very late in the course of the patient's cancer. A recent study showed that less than $5 \%$ of patients admitted to the hospital for an acute medical event had a documented advanced care plan (ACP). Although those numbers are higher for the elderly (12.6\% of those over 90) and those in institutional care (23.3\%), they still fall short of an objective that all adults document their ACP (Knight et al., 2020). Even in cancer patients, $40 \%$ had not discussed their EOL care preferences prior to death (Narang et al., 2015).

Clearly, ACP, GOC, and EOL conversations have, heretofore, been a small part of the patient's overall care plan and improvement is clearly needed. Such conversations are more important than ever in the current COVID-19 (SARS-CoV-2) pandemic (Lancet Editorial, 2020). Patients most at risk of dying from COVID-19 infection are elderly with underlying co-morbidities. Cancer, especially advanced stage incurable cancer, is one of the most significant co-morbidities (Dai et al., 2020). And patients with advanced cancer often have other chronic medical conditions such as tobacco-related cardiopulmonary disease, hypertension, diabetes, and organ dysfunction such as chronic kidney disease. Cancer patients have a threefold increase in the death rate from COVID-19 when compared with those without cancer and have much more severe disease with higher rates of intensive care unit (ICU) admission and mechanical ventilation (Dai et al., 2020).

As a result of this grim reality, now it is an important time to re-assess the approaches to GOC discussions (Lancet Editorial, 2020). A renewed emphasis on having these conversations early in the course of the patient's disease is necessary. In many cases, such conversations can be well received by patients and their families, especially when done early and in a calm setting where clear discussions and acknowledgement of goals can be addressed without the anxiety and distress that accompanies urgent decisions at the bedside of an imminently dying patient. 
One such approach was developed at MD Anderson Cancer Center, with a coordinated multi-disciplinary team established in March, 2020, in anticipation of the surge of the coronavirus pandemic in the USA. This team comprised oncologists, Division and Department Chairs, hospitalists, social workers, and specialists in Critical Care and Palliative Medicine. All patients admitted to the hospital were assessed for COVID-19 exposures and/or symptoms and, if they triggered the need for testing, were placed in a sequestered unit as a Patient under Investigation. Patients testing positive for COVID-19 were placed in a separate unit and managed by a single hospitalist team. As part of their admission, the patient's primary outpatient oncologist did a video visit with the patient and family to discuss GOC, EOL, and ACP as appropriate. This entailed a candid discussion about not only the prognosis of their underlying cancer but also the added impact of COVID-19 infection. Patients, admitted to the ICU for any reason, were also counseled and then assigned a risk score based on curative potential of their underlying cancer and co-morbidities. To make the conversations less daunting, institutional leaders in the Palliative Care Department provided numerous web-based tutorials on how to have such discussions and provided resources for clinicians to continue to hone their skills as needed. Many of these patient/ family encounters done in this fashion often resulted in a mutually agreed Do-Not-Resuscitate status and clarity regarding the role of cardio-respiratory support medications and equipment such as ventilators.

The result of this initiative not only addressed the acute issues but also helped highlight the importance of candid conversations in cancer patients at all points of their disease, from diagnosis to death. At MD Anderson Cancer Center, it has been policy since September, 2016 that "advance directive (AD) discussions should be held for new patients with a diagnosis code for invasive malignancy by the third office visit." Currently, Departmental Chairs are developing processes to measure ACP documentation for all patients in the outpatient setting with policy to update these documents as the patient's cancer status and/or treatments change. Although "a pandemic is a cause and powerful amplifier of suffering, through physical illness and death, through stresses and anxieties, and through financial and social instability" (Lancet Editorial, 2020), it can also help clinicians connect with their patients on these GOC issues earlier and provide a method by which these discussions are more easily undertaken.

\section{Conflict of interest}

There are no conflicts of interest.

\section{References}

Ambuel B (2001) Breaking bad news and discussing death. Primary Care 28, $249-267$.

Back AL (2005) Approaching difficult communication tasks in oncology. CA: A Cancer Journal for Clinicians 55, 164-177.

Baile WF (1999) Communication skills training in oncology. Description and preliminary outcomes of workshops on breaking bad news and managing patient reactions to illness. Cancer 86, 887-897.

Dai M, Liu D, Liu M, et al. (2020) Patients with cancer appear more vulnerable to SARS-CoV-2: A multicenter study during the COVID-19 outbreak. Cancer Discovery 10, 783-791.

Heyland DK (2006) What matters most in end-of-life care: Perceptions of seriously ill patients and their family members. CMAJ: Canadian Medical Association Journal 174, 627-633.

Knight T, Malyon A, Fritz Z, et al. (2020) Advance care planning in patients referred to hospital for acute medical care: Results of a national day of care survey. EclinicalMedicine 19, 100235.

Lancet Editorial (2020) Palliative care and the COVID-19 pandemic. The Lancet 395, 1168.

Lo B, Quill T and Tuksky J (1999) Discussing palliative care with patients. Annals of Internal Medicine 130, 744-749.

Marcus JD and Mott FE (2013) The final good-bye: Conversations at the end of life. Journal of Palliative and Supportive Care 11, 277-279.

Marcus JD and Mott FE (2014) Difficult conversations: From diagnosis to death. The Ochsner Journal 14, 712-717.

Marcus JD, Elkins G and Mott FE (2006) Communicating: Again and again. Supportive and Palliative Cancer Care 2, 37-41.

Mott FE and Adams KC (2019) Advance care planning and end-of-life issues in head and neck cancer. Journal of Palliative Care 34, 18-20.

Narang AK, Wright AA and Nicholas LH (2015) Trends in advance care planning in patients with cancer: Results from a national longitudinal survey. JAMA Oncology 1, 601-608.

Reddy S, Tanco K and Yennu S (2019) Integration of a mandatory palliative care education into hematology-oncology fellowship training in a comprehensive cancer center: A survey of hematology oncology fellows. Journal of Oncology Practice 15, e934-e941.

Rosenbaum ME (2004) Teaching medical students and residents skills for delivering bad news: a review of strategies. Literature Review in Academic Medicine 79, 107-117.

Singer PA, Martin D and Klener M (1999) Quality endof-life care: Patients' perspectives. Journal of the American Medical Association 281, 163-168.

Temel JS, Greer JA, Muzikansky A, et al. (2010) Early palliative care for patients with metastatic non-small-cell lung cancer. New England Journal of Medicine 363, 733-742.

Van de Kieft GK (2001) Breaking bad news. American Family Physician 64, 1975-1978.

Wong A, Reddy A and Williams JL (2016) Attitudes, beliefs, and awareness of graduate medical education trainees regarding palliative care at a comprehensive cancer center. Journal of Oncology Practice 12, e127-e137. 